\title{
Design and Fabrication of Polydimethylsiloxane Based Low Cost Microfluidic for Ultra-Fast Diagnostic Applications
}

\author{
S. R. Balakrishnan ${ }^{* 1,2}$, U. Hashim ${ }^{2}$, M. Sahrim ${ }^{1}$, I. Ismail ${ }^{1}$, J. Jamaludin ${ }^{1}$,

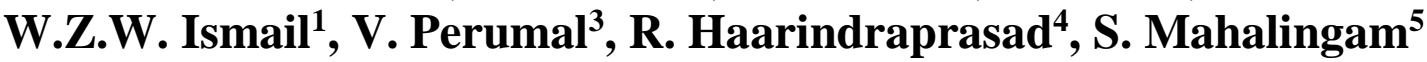

${ }^{1}$ Faculty of Engineering and Built Environment, Universiti Sains Islam Malaysia (USIM), Bandar Baru Nilai, 71800,
Nilai, Negeri Sembilan, Malaysia.
${ }^{2}$ Institute of Nano Electronic Engineering, University Malaysia Perlis (UniMAP), 01000 Kangar, Perlis, Malaysia.
${ }^{3}$ Centre of Innovative Nanostructure \& Nanodevices, Universiti, Teknologi PETRONAS, 32610 Bandar Seri Iskandar,
Perak Darul Ridzuan, Malaysia.
${ }^{4}$ Faculty of Applied Science, Department of biotechnology, AIMST University, Jalan Bedong-Semeling,
08100, Bedong, Kedah
${ }^{5}$ Institute of Sustainable Energy (ISE), Universiti Tenaga Nasional, Jalan IKRAM-UNITEN, 43000 Kajang, Selangor,
Malaysia.

Received 16 November 2017; accepted 6 June 2018, available online 6 August 2018

\begin{abstract}
Diagnostic tools such as Point-of-Care (POC) analysis usually requires an on-site testing and measurements. Hence, to ease the fluid sample handling, microfluidics technology has been opted frequently to be incorporated with lab-on-chip (LOC). Therefore, an easy and conventional method of design and fabrication of the microfluidic device implemented. In this study, the device is fabricated with Polydimethylsiloxane (PDMS) and SU8 using soft lithography technique. Herein, we focus on fluid transportation which is applied in clinical diagnostics and the transfer rate to the desired position as well as the fluid leakage during the transportation. Hence, the microfluidic sample transport channeling provides a practical solution for POC technological advances to study the biological systems that yield a better result in quantitative measurements and providing researchers new tools for life science research.
\end{abstract}

Keywords: Lab-on-Chip, Microfluidic, PDMS, SU-8, Point-of-Care.

\section{Introduction}

The domain of microfluidics technology is the backbone of wide variety of embodiments and applications which has conquered the field of biotechnology and engineering. For that reason, the inspiration of its use in biomedical and life science research can be focused into two areas [1-3]. One of the reason is the size of the device itself is getting small which is the current trend in engineering. While another key inspiration point is the use of the small sized device that makes it ideal platform for portable and point-of-care diagnostic devices. In spite being small, microfluidic devices are easy to use, cheap to fabricate and operate, require very little sample, and they can be easily disposed. For these reasons, the handheld diagnostic device has been recognized as a potential killer application of microfluidic and is the inspiration for much of the research effort in microfluidic technology development.

Advancements in microfluidic technology has realized the advantage of Lab-on-Chip (LOC) systems for analyzing small samples sizes to achieve the complex laboratory functions in biological applications [4]. Recently, considerable attention has been paid to point-ofcare (POC) diagnostic which can be performed at the bedside or in the clinic[2], [5], [6]. The realization of POC requires not only fast, sensitive and selective detection, but also small, cheap and integrated device [7]. The used method is known as in vitro diagnostic (IVD), which does not involve the use of laboratory staff and facility to provide the result. Besides, advancements in microchip fabrication technology provides convenience in integrating IVD to transmit results wirelessly, which could connect the patient and medicals in distance. As the result, these miniature LOC devices that made portable as POC diagnostic tools are currently dominating the pharmaceutical and health care market. This point has been made clear by the Global Industry Analysts (GIA), that by the year 2015, US\$12 billion revenue is generated by POC industry [8]. With that, application of nanotechnology, promises fast, ultra-high sensitive method to measure signal transduced from biological elements [9]. These devices are capable of analyzing the activity of biological systems which can be applied in many areas such as disease detection [10], clinical diagnosis, environmental quality monitoring [11] and food processing [12].

Up to now, several methods have been demonstrated for the fabrication of microfluidic, including polydimethylsiloxane (PDMS) [13], SU-8 [14], polymethylmethacrylate (PMMA) [15], waveguide pattern 
[16] and paper based [17]. PDMS and PMMA are often the best choices to fabricate microfluidic devices since it is cheap to construct, easy handling, non-toxicity, disposable because of its polymerization characteristics and multifunctional instead of glass and quartz based microfluidic [18]. Paper based microfluidic devices are also commonly used and extensively investigated in numerous studies [17]. Then, waveguide based microfluidic devices more likely to have disadvantages too, which the transmission of signals could be affected based on the waveguide shape [19].

Using PDMS and SU-8 (negative photoresist) is the most known procedure in fabrication of different microfluidic which known as soft lithography method. SU8 typically used because of its high aspect ratio employs as a master for molding and the PDMS accounts as a molding material that should be cured after pouring on the master [10]. High-aspect-ratio SU-8 molds for the fabrication of PDMS based microfluidic devices has been reported in numerous researches [20]. Devices fabricated form PDMS have proved to be more versatile and popular in many analytical applications [21]. Hydrophobicity of a surface is critical for protein attachment in the device [22]. The manipulation of surface properties ranging from hydrophobic to hydrophilic, charged or uncharged, smooth to rough, are important in defining biocompatibility [21]. In this paper we present a simple and inexpensive method to fabricate microfluidic based microchannels on oxide grown silicon wafer in order to achieve smooth and deep microchannels [23]. By using PDMS and SU-8 cast and peel method, the proposed design is fabricated under cleanroom environment. This covers the preliminary study to develop LOC device that practices microfluidic based fluid transport. In addition, the fluid flow and leakage is also analyzed by dropping aqueous solution into the inlet of the channel.

\section{Experimental Setup}

\subsection{Material, Design and Specifications}

The disposable PDMS based microfluidic module, which is the upper part of the LOC device, is first designed using AutoCAD software tool. It is a convenient tool for low cost mask design which is readable by multiple translators and able to convert it into industry standard mask making formats. The design of the microfluidic module is idealized based on fluid separation concept as in Figure 1(a); therefore, the final design made up of an inlet for fluid flow through a channel which eventually separated into three individual channels that lead to outlets. The designed microfluidic module is sent for outsourced chrome mask fabrication. Once the mask is prepared as in Figure 1(b)-(c), it can produce 20 individual die modules in a single $100 \mathrm{~mm}$ silicon wafer. From the specification illustrated in Figure 2, the microfluidic has an inlet and three outlets for fluid flow through each channel. The length is $12 \mathrm{~mm}$ and the width is $20.21 \mathrm{~mm}$ which makes it smaller than thumb size. Further, the radius of the inlet is $1.58 \mathrm{~mm}$, which is suitable for a drop of sample. The fluid outlets are much smaller than the inlet at $1.31 \mathrm{~mm}$ radius. Whereas, the dimensions of the microfluidic arc length measured at $6.31 \mathrm{~mm}$ outwards and $5.47 \mathrm{~mm}$ inwards. To ensure the capillary effect is enhanced the channel width were designed at $0.5 \mathrm{~mm}$ for a fast fluid transport to the biochip.
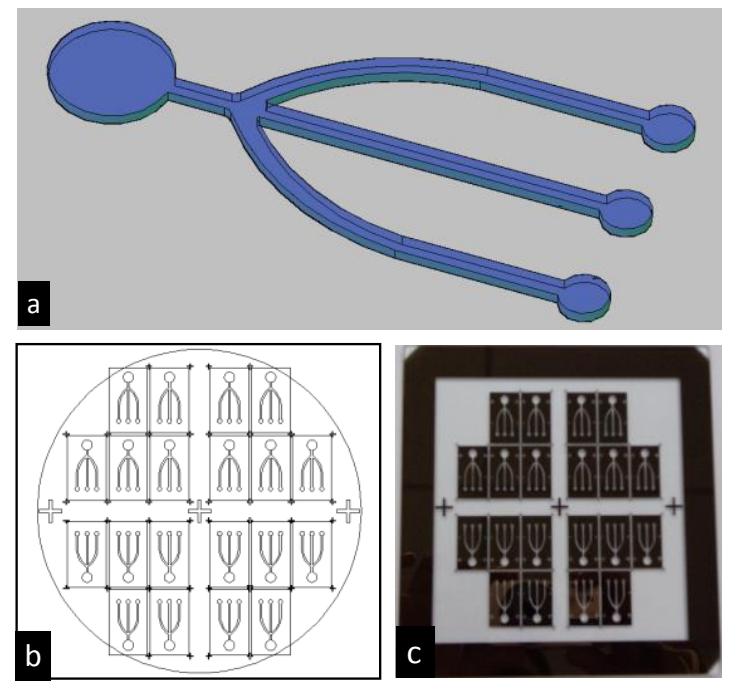

Fig. 1: The mask design and specifications of the microfluidic; (a) The designed microfluidic channels in AutoCAD. (b) Schematic design of microfluidic mask (c) The fabricated chrome mask design.

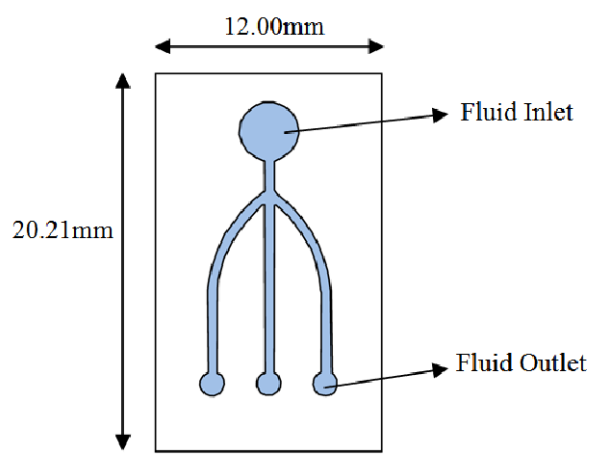

Fig. 2: Design and specifications of the microfluidic.

\subsection{Soft Lithography Fabrication}

The process flow of fabricating the SU-8 microstructure is briefly illustrated in Figure 3. The $\mathrm{SiO}_{2}$ grown wafer substrate is first cleaned in acetone and DI water. Then, the negative photoresist of SU-8 (MicroChem, USA) is spin-coated onto the substrate to form $300 \mu \mathrm{m}$ thick layer. It is then followed by soft-bake at $65^{\circ} \mathrm{C}$ for $10 \mathrm{~min}$ and at $95^{\circ} \mathrm{C}$ for $30 \mathrm{~min}$. A conventional lithography process of exposure dose of $600 \mathrm{~mJ} \mathrm{~cm}^{-2}$ is performed followed by a post-exposure bake process at $65^{\circ} \mathrm{C}$ for $3 \mathrm{~min}$ and at $95^{\circ} \mathrm{C}$ for $10 \mathrm{~min}$. As the finishing step, the exposed resist is developed and cured which results a microstructured SU-8 casting module template as in Figure 5 (a). In Figure 4, the process steps for PDMS moulding is shown. A mixture in specific ratio (10:1) of silicone elastomer and elastomer curing agent (Sil-More 
Industrial Ltd, USA Sylgard 184A and Sylgard 184B) is poured onto the SU-8 microstructure mold. The moulding is then cured at $70^{\circ} \mathrm{C}$ overnight and then finally cut and peeled off as seen in Figure 5 (b)-(c).

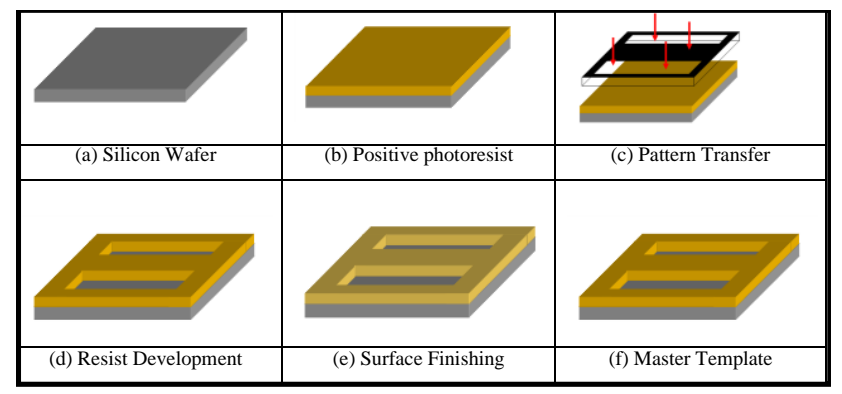

Fig. 3: Master Template Fabrication process flow.

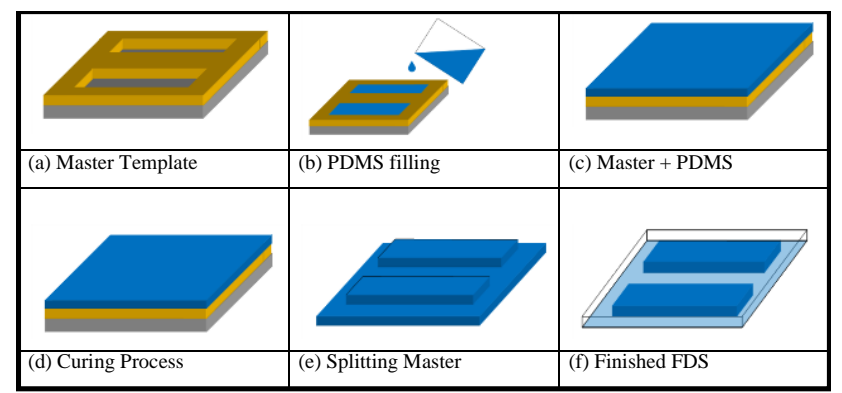

Fig. 4: PDMS microfluidic layer moulding process flow.
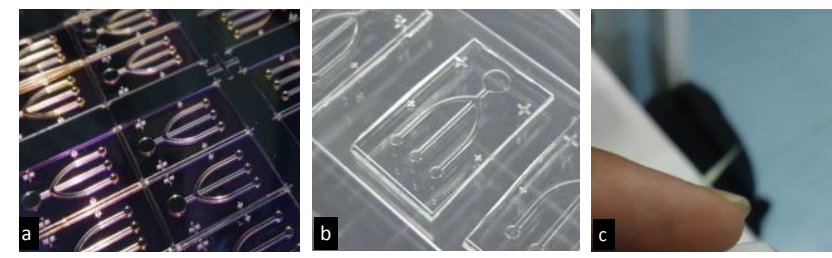

Fig. 5: PDMS layer moulding process flow. (a) The fabricated SU-8 microstructure template. (b) The moulded PDMS layer with master template. (c) The size and elasticity of the PDMS based microfluidic.

\section{Result and Discussions}

\subsection{Fluidic Channel Fabrication}

In the microfluidic fabrication method presented here, the fabrication quality was analysed based on the resist development process by using high power microscope as shown in Figure 6. From the observation, the SU-8 resist microstructure profile is clearly shows the difference in resist development. Figure 6 (a) shows an underdeveloped profile and partially developed profile in Figure 6 (b), (c) and (e) which is the undesired results. This non-developed profiles might be due to low etching time and this would further cause imperfection in the microfluidic channel depth. Whereas, the resist profile in Figure 6 (d)-(f) shows fully developed structure that occur due to the correct etching time and thus, yields a suitable surface for PDMS moulding. Hence, the PDMS layer that is filled in SU-8 mould, could be easily removed after the curing process.
This is due to the low surface energy characteristics of the PDMS which is realized by controlling the curing time. Besides that, the resulting shape of the microfluidic layer agrees well with the SU-8 mould that has been hardened to realize the shape. Here, the process parameters that applied is at 30 min curing under $65^{\circ} \mathrm{C}$.

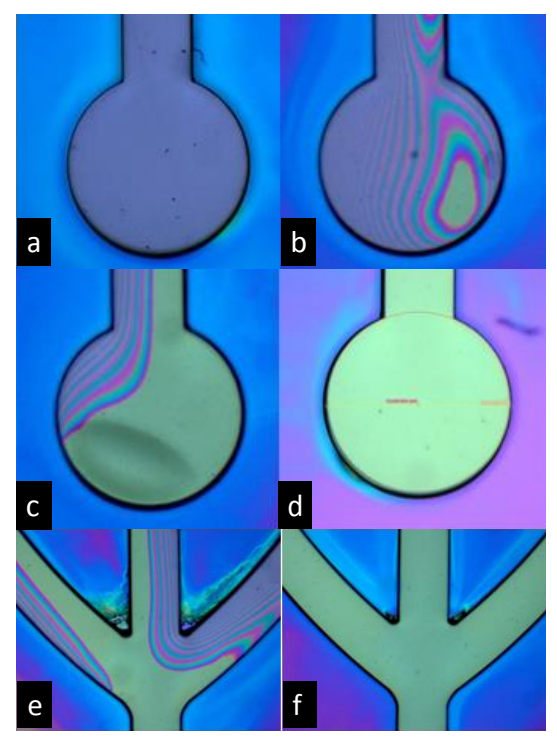

Fig. 6: The master template soft-lithography fabrication process flow. An underdeveloped (a), partially developed (b, c and e) and fully developed (d and f) SU-8 microstructure.

\subsection{Microfluidic Channel and Flow Analysis}

The inspection of the PDMS microfluidic device were also carried out morphologically to observe the channel depth and irregularities. Figure 7 (a)-(b) shows the channel depth and width inspected with high power microscope. The channel depth is measured at $300 \mu \mathrm{m}$. With this small depth of the connecting microchannel and since PDMS is a good elastomer, it is very easy to seal this microchannel during the bonding step by applying proper pressure onto this region. Figure 7 (c)-(d) depicts the leakage test after (c) and before (d) plasma bonding. It is noticed that before bonding the fluid has undergo leakage when mounted onto a glass slide. The bonding causes the PDMS microfluidic layer to bond firmly on the glass slide, and further reducing the hydrophobicity characteristics which causes leaking. For a better result, adhesive based or liquid PDMS pre-polymer could be applied to all the side ends of the bonded device followed by sufficient curing (UV and/or thermal curing) for better hermetic seal. Due to its high hydrophobicity, PDMS absorbs some organic solvents and some hydrophobic analytes, causing fouling of the material. These disadvantages greatly limit the variety of analytes that can be separated on PDMS devices, and generally results in relatively lower separation efficiency [18]. The fluid flow rate into the microfluidic channels were also studied as shown in Figure 8. Small sized holes were punctured through the inlet and outlets to make sure fluid flow in uninterrupted. In Figure 8 it is 
observed that the fluid takes less then 1 second to reach the outlet and this further shows the fabricated microfluidics functions as desired and fluid flow based on the capillary effect which does not requires further pressure to flow the liquid. Since, the research does not involve any surface modification on microchannels and fabricated under micro-scale technology, the usage of PDMS is an outstanding choice for disposable devices.

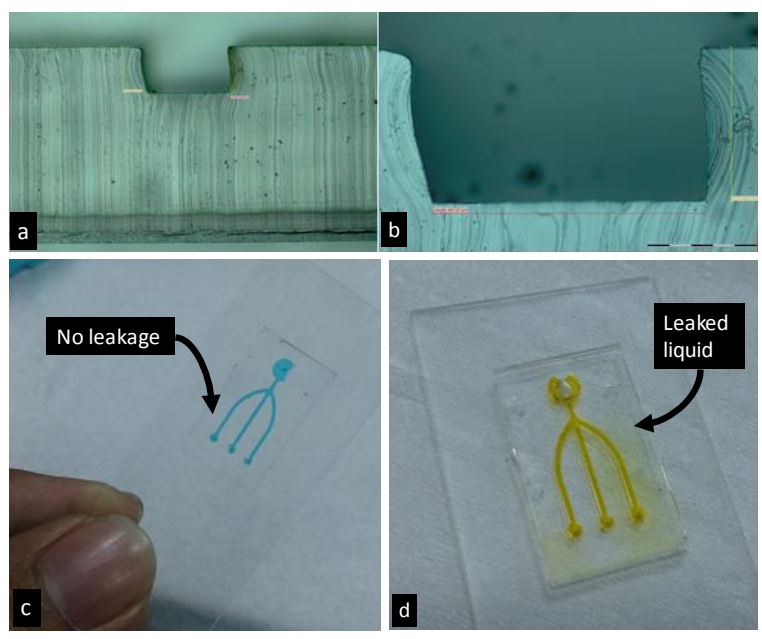

Fig. 7: Morphological channel depth and leakage analysis of the microfluidic.
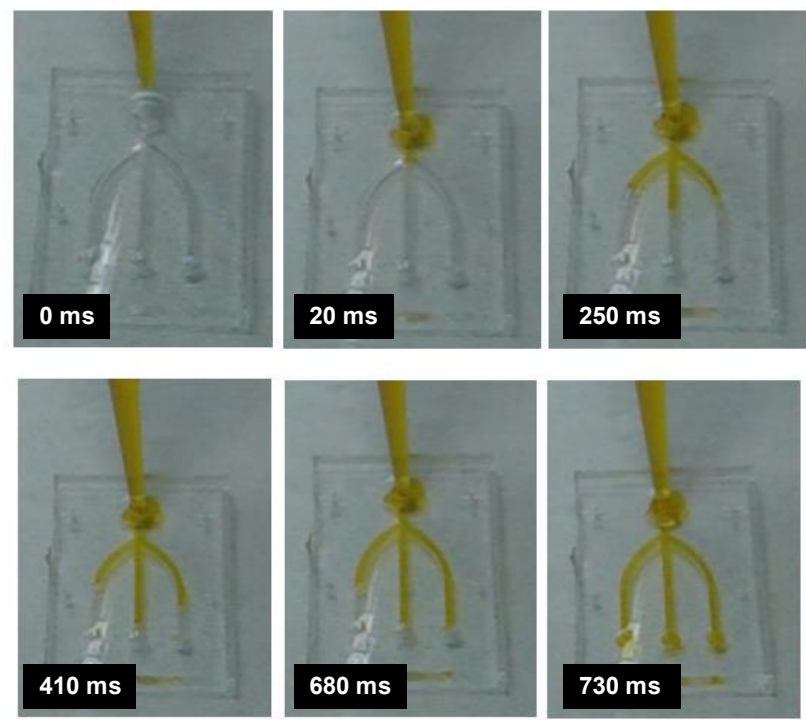

Fig. 8: Fluid flow analysis using coloured water to test the leakage and flow. A liquid resevoir is attacted to the inlet to allow the fluid flow.

\section{Conclusion}

The microfluidic design and fabrication analysis allowed us to choose dimensions and materials for the microstructure fabrication. Microfluidic devices using this fabrication approach must therefore be careful to assure that the desired operation matches the real functionality of the device. Since, SU-8 and PDMS are widely used in microfluidic fabrication, it is proven that this method is suitable and cheap.

\section{Acknowledgement}

The author thankfully acknowledges the staff at the Cleanroom Microfabrication Lab and Institute of Nano Electronic Engineering (INEE) at University Malaysia Perlis (UniMAP) for all their guidance and advice. The authors would like to thank Ministry of Higher Education Malaysia for the financial support through MTUN-COE grant 9016-00004. This work was supported by INEE at UniMAP, through the Biomedical Nano Diagnostics project.

\section{References}

[1] A. M. Streets and Y. Huang, "Chip in a lab: Microfluidics for next generation life science research," Biomicrofluidics, vol. 7, no. 1, p. 11302, 2013.

[2] S. Rao et al., "Polysilicon Nanogap Lab-on-Chip Facilitates Multiplex Analyses with Single Analyte," Biosens. Bioelectron., vol. 84, pp. 44-52, 2015.

[3] S. R. Balakrishnan et al., "Development of highly sensitive polysilicon nanogap with APTES/GOx based lab-on-chip biosensor to determine low levels of salivary glucose," Sensors Actuators, A Phys., vol. 220, pp. 101-111, Oct. 2014.

[4] A. R. Bahadorimehr, Y. Jumril, and B. Y. Majlis, "Low cost fabrication of microfluidic microchannels for Lab-On-a-Chip applications," in 2010 International Conference on Electronic Devices, Systems and Applications, ICEDSA 2010 Proceedings, 2010, pp. 242-244.

[5] S. R. Balakrishnan et al., "A Point-of-Care Immunosensor for Human Chorionic Gonadotropin in Clinical Urine Samples Using a Cuneated Polysilicon Nanogap Lab-on-Chip," PLoS One, vol. 10, no. 9, p. e0137891, 2015.

[6] S. C. B. Gopinath et al., "Nanogapped impedimetric immunosensor for the detection of 16ákDa heat shock protein against Mycobacterium tuberculosis," Microchim. Acta, pp. 1-7, 2016.

[7] V. Gubala, L. F. Harris, A. J. Ricco, M. X. Tan, and D. E. Williams, "Point of care diagnostics: Status and future," Anal. Chem., vol. 84, no. 2, pp. 487-515, Jan. 2012.

[8] G. I. A. Inc., "Biosensors in Medical Diagnostics - a Global Strategic Business Report,” 2012.

[9] M. Ferrari, BioMEMS and biomedical nanotechnology, vol. 4. Springer, 2007.

[10] W. Lee, J. Lee, and J. Koh, "Development and applications of chip calorimeters as novel biosensors," Nanobiosensors Dis. Diagnosis, vol. 1, pp. 17-29, 2012.

[11] N. B. Ramírez, a. M. Salgado, and B. Valdman, "The evolution and developments of immunosensors for health and environmental monitoring: Problems and perspectives," Brazilian J. Chem. Eng., vol. 26, no. 2, pp. 227-249, 2009. 
[12] J.-Y. Yoon and B. Kim, "Lab-on-a-Chip Pathogen Sensors for Food Safety," Sensors, vol. 12, no. 12, pp. 10713-10741, Jan. 2012.

[13] Z. Almutairi, C. L. Ren, and L. Simon, "Evaluation of polydimethylsiloxane (PDMS) surface modification approaches for microfluidic applications," Colloids Surfaces A Physicochem. Eng. Asp., vol. 415, no. 2010, pp. 406-412, Dec. 2012.

[14] C. De Azurem, H. C. Oliveira, J. G. Rocha, I. Barros, J. M. Cabral, and G. Minas, "SU-8 microfluidic mixer for use in lab-on-a-chip devices for biological fluids analyses," in Proceedings of the IEEE International Conference on Industrial Technology, 2006, pp. 3014-3019.

[15] H. Y. Tan, W. K. Loke, and N.-T. T. Nguyen, "A reliable method for bonding polydimethylsiloxane (PDMS) to polymethylmethacrylate (PMMA) and its application in micropumps," Sensors Actuators B Chem., vol. 151, no. 1, pp. 133-139, Nov. 2010.

[16] H. Andersson and A. Van den Berg, "Microfluidic devices for cellomics: A review," Sensors Actuators, B Chem., vol. 92, no. 3, pp. 315-325, 2003.

[17] X. Li, D. R. Ballerini, and W. Shen, "A perspective on paper-based microfluidics: Current status and future trends," Biomicrofluidics, vol. 6, no. 1, pp. 12 14, Mar. 2012.

[18] R. Liang, P. Hu, G. Gan, and J. Qiu, "Deoxyribonucleic acid modified poly(dimethylsiloxane) microfluidic channels for the enhancement of microchip electrophoresis," Talanta, vol. 77, no. 5, pp. 1647-1653, Mar. 2009.

[19] A. Prabhakar and S. Mukherji, "C-shaped embedded polymer waveguide for evanescent field absorption based lab on a chip biosensor," in Systems in Medicine and Biology (ICSMB), 2010 International Conference on, pp. 67-70.

[20] X. Chen, L. Zhang, H. Li, J. Sun, H. Cai, and D. Cui, "Development of a multilayer microfluidic device integrated with a PDMS-cellulose composite film for sample pre-treatment and immunoassay," Sensors Actuators A Phys., vol. 193, pp. 54-58, Apr. 2013.

[21] K. Liu, Y. Tian, R. Pitchimani, M. Huang, H. Lincoln, and D. Pappas, "Characterization of PDMSmodified glass from cast-and-peel fabrication," Talanta, vol. 79, no. 2, pp. 333-338, Jul. 2009.

[22] V. Anand, S. Ghosh, M. Ghosh, G. M. Rao, R. Railkar, and R. R. Dighe, "Surface modification of PDMS using atmospheric glow discharge polymerization of tetrafluoroethane for immobilization of biomolecules," Appl. Surf. Sci., vol. 257, no. 20, pp. 8378-8384, Aug. 2011.

[23] B. Sharma Rao and U. Hashim, "Microfluidic Photomask Design Using CAD Software for Application in Lab-On-Chip Biomedical Nanodiagnostics," Adv. Mater. Res., vol. 795, pp. 388-392, 2013. 